\section{Leadership for patient safety}

\section{THE ESTABLISHMENT OF A RISK AND SAFETY COMMITTEE IN A PRIVATE HEALTHCARE ORGANISATION}

Penny Kechagioglou*, Robert Chaldecott. GenesisCare UK

10.1136/leader-2020-FMLM.108

Background A culture of patient safety in Healthcare ensures that patients are treated right every time. To develop and sustain a patient safety culture requires a whole system approach and the role of clinical leaders is key.

Methods Our organisation's Leadership team audited all incidents weekly between September 2018 and January 2019 and found variable incident reporting rates amongst our clinical facilities. Lessons learned from incidents were not well cascaded to front-line teams. A Just Culture framework tool was piloted between January and August 2019 in an effort to improve incident reporting rates. This led to the establishment of a weekly multidisciplinary Risk and Safety Committee in October 2019, led by a Regional Physicist with expertise in Quality and Safety and the Medical Director. The RSC members represented front line teams, middle managers and the UK Leadership team. The weekly RSC facilitated risk analysis and the timely and accurate completion of Root Cause Analyses (RCA) by implementing a Just Culture policy.

Results The clinically led Risk and Safety Committee was established as a multidisciplinary forum where people felt safe to discuss healthcare incidents. The SBAR (Situation, Background, Analysis, Recommendation) reporting tool and the 5 Why/A3 process RCA tool were used to support a deep dive into root causes. An RCA registry with actions and lessons learned was established which facilitated the cascade of information to all teams. After six months of being operational, there have been 300 quality improvement actions documented by the RSC, $90 \%$ of which have been effected successfully.

Conclusions The successful implementation of the Risk and Safety Committee in our organization was the result of clinical and non-clinical leaders working together and learning from incidents. The culture of patient safety has improved, we see better incident reporting rates and people feel empowered to make quality improvement changes.

\section{Developing effective leaders}

\section{IMPROVING WELLBEING THROUGH PEER TO PEER SUPPORT}

Salma Eltoum Elamin*, David Cussons. Salisbury NHS Foundation Trust, Salisbury, Wiltshire, UK

\subsection{6/leader-2020-FMLM.109}

Background This project was aimed at addressing junior doctors' wellbeing in a district general hospital. It was initiated and delivered as part of a leadership initiative supported by the Wessex Deanery (Wessex Chief Resident Programme 2019-2020).
Burn out rates among doctors range between 25\% and $76 \%$. The peer to peer support interventions are designed to improve coping and resilience among doctors and breaking the burnout cycle. The aim of this project was to evaluate the effect of peer to peer support groups in junior doctors' morale and well-being. The peer-to-peer support provision as an intervention was introduced as an adjunct to routine trainee support measures already in place, i.e., educational programme and mentoring support.

Method The peer-to-peer support meetings were organised through an opt in approach. All participants and the facilitator agreed on terms of reference for the meetings including confidentiality. Participants completed a feedback survey investigating how the sessions affected their clinical practice and wellbeing. No ethical approval was sought for this project as it was not considered to the needed.

Results Twenty-three junior doctors participated in 20 sessions delivered over 6 months period. $25 \%$ completed a feedback survey. One third of the participants attended more than $70 \%$ of the sessions. Most participants scored 5 or more on the Likert scale for whether the sessions have changed their: sense of well-being at work, confidence levels, communication with patients and management of difficult situations.

Recommendation This project provides further evidence that peer-to-peer support is beneficial for junior doctors' wellbeing and morale. Positive participant feedback has encouraged other staff members to express interest in participation in a similar exercise. Dissemination plans are underway. It also provides further evidence that encouraging junior doctors to undertake leadership initiatives is transformative.

\section{Leading innovation and improvement}

\section{PATIENT ACCEPTABILITY OF TELEPHONE FOLLOW UP AFTER CATARACT SURGERY}

${ }^{1}$ Sarah Khavandi* ${ }^{*}$ Ernest Lim ${ }^{2,3},{ }^{3,4}$ Guy Mole. ' Oxford NHS Foundation School, Oxford, UK; ${ }^{2}$ Oxford University Hospitals NHS Foundation Trust; ${ }^{4}$ Ufonia Limited, Oxford, UK; ${ }^{3}$ Imperial College Healthcare Trust, London, UK

\subsection{6/leader-2020-FMLM.110}

GM and EL are both employees of Ufonia Ltd a company funded by Innovate UK that specialises in automating routine healthcare using natural language processing over the telephone.

Aims Healthcare is often slow to adopt innovation due to difficulties in changing culture and attitudes towards technological alternatives. This study aimed to evaluate the patient acceptability of a novel method for providing cataract follow up over the telephone (TFU) by looking at multiple factors of acceptability. This methodology can be used as a reproducible framework to guide co-development of future technology such as automated artificial-intelligence (AI) enabled TFU.

Methods Patients between the ages of 66 and 90 were called 3 weeks after their surgery by an ophthalmologist to assess if they had symptoms that could indicate a complication and require face-to-face review. We interviewed 30 consecutive patients who had received TFU within the last month. Patient acceptability was assessed through the validated Telehealth Usability Questionnaire (TUQ) and the likert scale responses 
were analysed. Qualitative data was collected through a semi structured questionnaire and a Braun and Clarke (2006) method of thematic analysis was carried out.

Results The majority of patients found that TFU post cataract operation was an acceptable way to receive follow up. On average patients scored $4.94 / 5$ on the likert scale for the simplicity and ease of use. Overarching themes that emerged were that TFU is more desirable as it saves time, travel and NHS resources. Overall, patients felt that if they had a postoperative complication they would have preferred to have seen a clinician in person. We found patients' views influenced by the COVID-19 pandemic meaning they saw additional benefits of telephone follow up compared to face-toface review.

Conclusions The majority of patients felt that TFU after cataract surgery was an acceptable way to receive healthcare. This has implications for how follow up is provided after the most common operation in the UK and worldwide and opens the possibility of this being provided by an automated AI driven system in the future.

\section{REDUCING INTRA-HOSPITAL TELEPHONE COMMUNICATION TIME USING APP TECHNOLOGY}

Mohammed Hamid. Royal Shrewsbury Hospital, UK

10.1136/leader-2020-FMLM.111

Background Lengthy switchboard waiting times result in delayed communication between healthcare professionals in a hospital. Wasted time impedes patient care, costs the Trust a substantial financial sum and impacts healthcare professional's morale. Aim: To reduce intra-hospital telecommunication time utilising the Induction phone application, an easy to use, regularly updated telephone directory. Methods: Initial audit: Five chosen specialities were contacted between 9-10 AM from the Emergency department for 2 consecutive weeks. The time taken to reach each speciality via switchboard was recorded. A survey seeking the number of calls made per day, the preferred method of contact and the feelings associated with telephone waiting times was sent to department doctors. PDSA cycle 1: One-month application advertisement and re-audit. PDSA2: Eye-catching tele-directory board with the most used extensions and bleeps displayed in the department. Satisfaction survey sent post PDSA2. Sustainability: New doctors were provided induction information. Results: Initial average waiting time via switchboard was 48 seconds. The average calls made per doctor each day was 12 . This calculated to a total departmental loss of 20.16 hours per week waiting on the phone, equating an annual loss of $£ 26,208$. PDSA1: Average waiting times reduced to 12 seconds utilising the application; saving an estimate $\sim £ 19,656$ per annum. PDSA2: Instant availability of contact details on the display board further reduced waiting times to an average 6 seconds. $84 \%$ of doctors $(n=16)$ disliked waiting more than 20 seconds, with associated feelings of frustration. $100 \%$ preferred the display board, then the use of the application before resorting to switchboard. 100\% Sustainability was recorded one year later. Conclusion: The use of application technology reduces wasted time which hampers patient care; reduces Trust running costs; and improves health care professional's morale at work.

\section{Understanding leadership through research}

\section{VILLAGES AGAINST CERVICAL CANCER: IMPROVING INDIA'S CERVICAL CANCER BURDEN BY EMPOWERING ASHA WORKERS}

1,2Sarayoo Ravishankar Vaidya. 'Independent researcher, MS Ramaiah Medical College Bengaluru, India; ${ }^{2}$ Masters in International Health student, Charité - Universitätsmedizin Berlin, Germany

\subsection{6/leader-2020-FMLM.112}

Cervical cancer is the second leading cause of new cancer cases and cancer-related deaths among women in India, with an estimated 96,922 new cases and 60,078 deaths each year. (ICCP,2020) India bears over a tenth of the global burden of cancers, with preventable cancers like cervical, oral and breast cancer forming more than a third of the disease burden.(Ferlay, 2015).

Currently, early diagnosis via low cost screening methods play the biggest role in curbing the burden of disease. The screening methods employed are colposcopy examination and routine pap smears every 5 years. However, both screening methods, while effective in identifying the disease, require skilled clinicians and laboratories with appropriate set-ups.

One method to circumvent this problem of low screening rates is to train rural local women to carry out Visual Inspection of cervix with Acetic acid (VIA) as a primary method of screening, as it is a low cost method of screening which does not require much laboratory facilities.

The aim is to scale up the current rate of screening of cervical cancer and subsequently decrease the case fatality rate of cervical cancer in Indian villages. This will be done by training Female health care workers and rural health practitioners to carry out Visual inspection of the Cervix.

Overall, a lot is left to be done to help improve the burden of cancer in India, but the best route is to start with harmony of Public and private sector and prioritization of cancer care in India.

There is also a need to improve connectivity between rural and urban areas to facilitate shunting of cancer patients for tertiary care. Coordination with Ministries of urban development, transportation and infrastructure developers is necessary to ensure this.

\section{Leadership lessons from across the world}

\section{TRAINING EMERGENCY MEDICAL STAFF TO APPROPRIATELY HANDLE PATIENTS WITH CHEST PAIN}

\footnotetext{
1,2Sarayoo Ravishankar Vaidya. 'Independent researcher, MS Ramaiah Medical College Bengaluru, India; ${ }^{2}$ Masters in International Health student, Charité - Universitätsmedizin Berlin, Germany
}

\subsection{6/leader-2020-FMLM.113}

Coronary artery disease(CAD) is a common cause of morbidity and mortality in the world and many patients with CAD present with chest pain in an emergency setting as a first presentation. In emergency settings in India, the burden of diagnosis and treatment of patients with chest pain often falls on the emergency physician alone, who also has to deal with 